\title{
Probing Dust Settling in Proto-planetary Disks with ALMA
}

\author{
Yann Boehler ${ }^{1,3, a}$, Anne Dutrey ${ }^{2, b}$, Stéphane Guilloteau ${ }^{3, c}$, and Vincent Piétu ${ }^{3, d}$ \\ ${ }^{1}$ Centro de Radioastronomı y Astrofisica, UNAM, Apartado Postal 3-72, 58089 Morelia, Michoacan, Mexico \\ ${ }^{2}$ Univ. Bordeaux, LAB, UMR 5804, F-33270, Floirac, France \\ ${ }^{3}$ CNRS, LAB, UMR 5804, F-33270 Floirac, France \\ ${ }^{4}$ IRAM, 300 rue de la Piscine, 38400 Saint Martin d'H'eres, France
}

\begin{abstract}
Investigating the dynamical evolution of dust grains in proto-planetary disks is a key issue to understand how planets should form. We identify under which conditions dust settling can be constrained by high angular resolution ALMA observations at $\mathrm{mm}$ wavelengths, and which observational strategies are suited for such studies. We find out that an angular resolution better than or equal to » 0.1 " (using $2.3 \mathrm{~km}$ baselines at $0.8 \mathrm{~mm}$ ) allows us to constrain the dust scale height and flaring index with sufficient precision to unambiguously distinguish between settled and non-settled disks, provided the inclination is close enough to edge-on $\left(i>75^{\circ}\right)$. Ignoring dust settling and assuming hydrostatic equilibrium when analyzing such disks biase the derived dust temperature, the radial dependency of the dust emissivity index and the surface density distribution.
\end{abstract}

\section{Introduction}

Grain growth and dust settling are two key ingredients in the planets formation process. Settling will speed up the growth process by favouring grain collisions, firstly by increasing the relative vertical velocities, as settling acts differently in function of the dust dynamic properties [1], and secondly by concentrating dust close to the midplane. On top of that, a high dust to gas ratio in this area, can affect the gravitational stability and control the initial step of the formation of planetesimals [2]. Contrary to other shortest wavelenghts, the moderate opacity of the $\mathrm{mm} / \mathrm{submm}$ domain allows dust mass estimate and should accordingly probe material throughout the complete disk structure. In this paper, we then study the impact of dust settling on the disk imaging at mm wavelengths, in order to define adequate observational strategies to constrain this phenomenon with ALMA.

\section{Methods}

We investigate here potential way to distinguish any settled disk from any non-settled one. Our approach is to analyze simulated images of settled disks with non-settled, homogeneous disk models. Under this approach, settled disks may result in very unusual parameters which cannot be ascribed

\footnotetext{
a e-mail: y.boehler@crya.unam.mx

be-mail: Mailaddressforsecondauthorifnecessary

$\mathrm{c}_{\mathrm{e}-\mathrm{mail}}$ : Mailaddressforlastauthorifnecessary

$\mathrm{d}_{\mathrm{e}-\mathrm{mail}}$ : Mailaddressforlastauthorifnecessary
} 
Table 1. Disk physical parameters for a 1 solar mass.

\begin{tabular}{c|c}
\hline \hline Physical characteristics & Adopted values \\
\hline type of grains & Moderate $(\leq 3 \mathrm{~mm})$ or Large $(\leq 10 \mathrm{~cm})$ \\
gas scale height & Hydrostatic Equilibrium \\
Averaged Gas/Dust & 100 \\
Kinetic and Dust Temperature & $T_{k}(r)=30\left(\frac{r}{R_{0}}\right)^{-0.4}$ Kelvin \\
Reference and Inner radius & $R_{0}=100 \mathrm{AU}$ and $R_{\text {int }}=3 \mathrm{AU}$ \\
\hline \multicolumn{2}{|c}{ Truncated disk } \\
\hline Gas Surface Density $\left(\mathrm{g} . \mathrm{cm}^{-2}\right)$ & $4.35\left(\frac{r}{R_{0}}\right)^{-1}$ with $R_{\text {out }}=100 \mathrm{AU}$ \\
\hline \multicolumn{2}{|c}{ Tapered disk } \\
\hline Gas Surface Density $\left(\mathrm{g} . \mathrm{cm}^{-2}\right)$ & $17.4\left(\frac{r}{R_{0}}\right)^{-0.5} \cdot \exp \left(\left(\frac{R}{R_{c}}\right)^{1.5}\right)$ with $R_{c}=50 \mathrm{AU}$ \\
\hline
\end{tabular}

to "normal" non-settled disks. For example, the dust scale height $H_{0}$ should be small, as well as the flaring index $h$, in comparison with the hydrostatic scale height.

\subsection{Disk Description}

The disks parameters are representative of the disks studied by [3] and are given in Table 1 . The disks are in hydrostatic equilibrium with no vertical temperature gradient and orbit around a $1 M_{\odot}$ star. In the truncated model, the gas surface density is a simple power law with sharp inner and outer radius. In the viscous model, the density is tapered by an exponential outer edge. Both models have the same total (gas+dust) disk mass is 0.03 solar mass. Each disk has been imaged at 4 inclination angles $\left(90^{\circ}\right.$, $85^{\circ}, 80^{\circ}$ and $\left.70^{\circ}\right)$.

\subsection{Dust Properties}

\subsubsection{Dust emissivity}

The dust emissivity as a function of frequency depends on the dust size distribution and grain composition. Given the important unknowns in the dust geometry and composition, we have elected to use a parametric method to model. Our dust emissivity model is based on two asymptotes: one at small wavelengths $(a \gg \lambda)$ where the absorption coefficient is dictated by the geometrical cross section, and another at long wavelengths $(a \ll \lambda)$ for which a power law applies. These two regimes are then connected by a resonance region near $\lambda=2 \pi a$, caracterized by its height and width.

The minimum grain size was $0.01 \mu \mathrm{m}$ and the maximum grain size $3 \mathrm{~mm}$ for the moderate grain model or $10 \mathrm{~cm}$ for the large grain model. The dust size distribution was represented by a power law of index $p_{d}=3$ and with the constant density $\rho_{d}=1.5 \mathrm{~g} . \mathrm{cm}^{-3}$. In the example presented in these proceedings, the parameters have been adjusted in order to match the dust properties used by [4].

\subsubsection{Dust settling}

For obtaining the dust settling, we decided to use an approximation based on the results of global numerical calculations derived from theoretical approaches [5] which take into account ideal MRIinduced MHD turbulence predictions [6,7] as well as vertical stratification of dust and gas. 
Instabilities and Structures in Proto-Planetary Disks

Table 2. Minimizations of a tapered settled disk by an homogeneous truncated one: moderate size grains

\begin{tabular}{|c|c|c|c|c|c|c|c|c|}
\hline $\begin{array}{l}\text { Disk Case \& } \\
\text { inclination }\end{array}$ & $\begin{array}{l}T_{0} \\
30\end{array}$ & $\begin{array}{c}q \\
0.4\end{array}$ & $p$ & $R_{\text {out }}$ & $\begin{array}{c}\beta_{i} \\
0.613\end{array}$ & $\begin{array}{c}H_{0} \\
14.6\end{array}$ & $\begin{array}{c}h \\
1.30\end{array}$ & $x^{2}$ \\
\hline $\begin{array}{l}\text { Assuming dust non-settling } \\
70^{\circ} \\
80^{\circ} \\
85^{\circ} \\
90^{\circ}\end{array}$ & $\begin{array}{l}34.8 \pm 0.07 \\
35.0 \pm 0.07 \\
29.5 \pm 0.13 \\
19.9 \pm 0.26\end{array}$ & $\begin{array}{l}0.319 \pm 0.001 \\
0.346 \pm 0.002 \\
0.504 \pm 0.004 \\
0.542 \pm 0.008\end{array}$ & $\begin{array}{c}2.14 \pm 0.002 \\
1.85 \pm 0.004 \\
1.31 \pm 0.006 \\
0.16 \pm 0.01\end{array}$ & $\begin{array}{l}103.0 \pm 0.03 \\
103.0 \pm 0.03 \\
109.0 \pm 0.06 \\
109.6 \pm 0.09\end{array}$ & $\begin{array}{l}0.442 \pm 0.001 \\
0.292 \pm 0.001 \\
0.293 \pm 0.003 \\
0.930 \pm 0.006\end{array}$ & $\begin{array}{l}(15.7) \\
(15.8) \\
(14.5) \\
(11.9)\end{array}$ & $\begin{array}{l}(1.34) \\
(1.33) \\
(1.25) \\
(1.23)\end{array}$ & $\begin{array}{l}1276606 \\
1415333 \\
1683039 \\
1319208 \\
\end{array}$ \\
\hline $\begin{array}{l}\text { Assuming dust settling } \\
70^{\circ} \\
80^{\circ} \\
85^{\circ} \\
90^{\circ}\end{array}$ & $\begin{array}{l}35.8 \pm 0.1 \\
35.5 \pm 0.06 \\
32.5 \pm 0.06 \\
29.2 \pm 0.13\end{array}$ & $\begin{array}{l}0.306 \pm 0.001 \\
0.306 \pm 0.003 \\
0.351 \pm 0.004 \\
0.294 \pm 0.012\end{array}$ & $\begin{array}{c}2.23 \pm 0.002 \\
1.95 \pm 0.005 \\
1.62 \pm 0.008 \\
0.20 \pm 0.01\end{array}$ & $\begin{array}{l}106 \pm 0.03 \\
109 \pm 0.03 \\
119 \pm 0.04 \\
113 \pm 0.06\end{array}$ & $\begin{array}{l}0.466 \pm 0.001 \\
0.390 \pm 0.001 \\
0.444 \pm 0.002 \\
0.754 \pm 0.076\end{array}$ & $\begin{array}{l}13.4 \pm 0.04 \\
7.7 \pm 0.04 \\
3.0 \pm 0.04 \\
4.0 \pm 0.04\end{array}$ & $\begin{array}{l}1.33 \pm 0.005 \\
1.21 \pm 0.008 \\
0.46 \pm 0.01 \\
0.10 \pm 0.02\end{array}$ & $\begin{array}{l}1262191 \\
1203651 \\
1156239 \\
1162383\end{array}$ \\
\hline
\end{tabular}
second row indicates the expected values of the parameters.

The main factor controlling the degree of settling is the dimensionless product of the dust stopping time $\tau_{s}$ by the angular velocity. This product is proportional to the grain size. When $\Omega \tau \ll 1$, the dust particles are coupled to the gas. On the contrary, when $\Omega \tau \gg 1$ (case for large grains), the dust particles are decoupled from the gas and settle towards the midplane.

It is convenient to further approximate the effects of dust settling by relating the "settling factor" $s(a, r)=H_{d}(a, r) / H_{g}(a, r)$ to the settling parameter $\Omega \tau_{0}=\left(\Omega \tau_{s}\right)(r, z=0)$ : We have adopted the following law, which matches the previous asymptotic results

$$
\begin{aligned}
s(a, r) & =1 & \text { if } & \Omega \tau_{0}<\omega_{c} \\
& =\left(\frac{\Omega \tau_{0}}{\omega_{c}}\right)^{-0.5} & \text { if } & \Omega \tau_{0}>\omega_{c}
\end{aligned}
$$

with $\omega_{c}=6.510^{-4}$ being equal to the viscosity parameter $\alpha$, within a factor of order unity [8].

\subsection{Disks Simulations}

We used the ray-tracer of the radiative transfer code DISKFIT [9] to generate brightness distributions at different wavelengths. The simulated brightness distributions obtained from DISKFIT were then processed through the regularly upgraded ALMA simulator implemented in the GILDAS software package [10] in order to produce the visibilities.

As a first guess, we choose to simulate observations obtained using 50 antennas with a single antenna configuration, so that observations at different wavelengths can be performed nearly simultaneously. A maximum baseline length of $2.3 \mathrm{~km}$ was used and the observations were assumed to be around the transit. Pseudo-observations of settled disks, have been created at four different frequencies, 100, 230, 340 and $670 \mathrm{GHz}$. At the distance of the nearest star forming regions $(140 \mathrm{pc}$ for Taurus-Auriga), the corresponding linear resolutions are 42, 18, 12 and 6 AU.

\section{Results}

Attempting to represent a settled disk by non-settled ones introduces several inconsistencies which reveal the existence of dust settling, provided the inclination is high enough. Table 2 displays the results.

\subsection{Deriving the Scale Height}

If we assume we are in hydrostatic equilibrium and that the dust is not settled, this hypothesis leads to unusual results. When viewed edge-on, the derived temperature is to some extent forced towards 
low values to better mimic the small disk thickness ( $\sim 19 \mathrm{~K}$ instead of $30 \mathrm{~K})$. Relaxing the non-settled hypothesis allows us to recover the input temperature profile. For the moderate grains models, we found at high inclinations a dust scale height of 3-4 AU and of 2-3 AU for the large grains. By comparaison to the gas scale height which is equal to about $14 \mathrm{AU}$, this discrepancy shows clearly the existence of the dust settling.

\subsection{Impact of the Surface Density Profile}

If the surface density law for the pseudo-observation between the pseudo-observation and the model is not exactly the same, the dust scale height is more difficult to derive at low inclination (i. e. $<75-80$ degrees). This result indicates that at inclinations below $80^{\circ}$, the recovered scale height is sensitive to the exact shape of the surface density distribution, and cannot in general be determined accurately. Moreover, settled disks actually appear "pinched" $(h<1)$ rather than flared $(h>1)$.

\subsection{Others parameters}

Although they can serve as secondary indicators and are sensitive to the dust settling at high inclination $\left(\geq 80^{\circ}\right)$, others parameters like the spectral index $\beta, p, \ldots$ cannot serve as diagnostics.

\subsection{Instrumental Effects:}

Thermal noise was added to the simulated $u v$ data (corresponding to $30 \mathrm{~min}$ of observations for each frequency). The resulting image noise (point source sensitivity) are $13 \mu \mathrm{Jy}$ at $100 \mathrm{GHz}, 20$ at 230 $\mathrm{GHz}, 30$ at $340 \mathrm{GHz}$ and 111 at $670 \mathrm{GHz}$ and did not prevent us to observe the dust settling. Thermal noise is then not a serious limitation to measure dust settling.

However, the atmosphere induces path length variations which are only partially recovered by the calibration techniques anticipated for ALMA. We have then evaluated the impact of the atmospheric phase noise and found that it should be below about $40^{\circ}$ to avoid smearing by limited seeing.

\subsection{Impact of the various Wavelengths}

The above studies show that all the impact of dust settling is only in the effective scale height. Then, if we know the gas structure from previous observations, only one frequency is necessary. The best frequency for our analysis, giving sufficient spatial resolution and low thermal and phase noise was $340 \mathrm{GHz}$. More generally, spatial resolutions lower than 1" (or 14 AU for the Taurus aurigae region), no longer have sufficient resolution for our propose.

Nevertheless, if the gas structure is unknown, we need at least two frequencies to derive the dust temperature by resolving the optically thick parts of the disk. With dust emissivity index radial gradients like found by [3] and predicted by simulations of [1], proper identification of an optically thick core region requires at least 3 frequencies.

\section{References}

[1] T. Birnstiel, L. Ricci, F. Trotta, C.P. Dullemond, A. Natta, L. Testi, C. Dominik, T. Henning, C.W. Ormel, A. Zsom, A\&A 516, L14+ (2010), 1006.0940

[2] P. Goldreich, W.R. Ward, ApJ 183, 1051 (1973)

[3] S. Guilloteau, A. Dutrey, V. Piétu, Y. Boehler, A\&A 529, A105+ (2011), 1103. 1296 
[4] L. Ricci, L. Testi, A. Natta, R. Neri, S. Cabrit, G.J. Herczeg, A\&A 512, A15+ (2010), 0912. 3356

[5] S. Fromang, R.P. Nelson, A\&A 496, 597 (2009), 0901.4434

[6] S.A. Balbus, J.F. Hawley, ApJ 376, 214 (1991)

[7] S.A. Balbus, J.F. Hawley, Reviews of Modern Physics 70, 1 (1998)

[8] B. Dubrulle, G. Morfill, M. Sterzik, Icarus114, 237 (1995)

[9] V. Piétu, A. Dutrey, S. Guilloteau, A\&A 467, 163 (2007), arXiv: astro-ph/0701425

[10] J. Pety, F. Gueth, S. Guilloteau, ALMA Memo 386, 1 (2002) 